\title{
TERT gene: its function and dysregulation in cancer
}

\author{
Andrew J Colebatch, ${ }^{1}$ Alexander Dobrovic, ${ }^{2,3,4,5}$ Wendy A Cooper ${ }^{\oplus 1,6,7}$
}

${ }^{1}$ Tissue Pathology and Diagnostic Oncology, Royal Prince Alfred Hospital, Sydney, Australia

${ }^{2}$ Translational Genomics and Epigenomics Laboratory, Olivia Newton-John Cancer Research Institute, Heidelberg, Victoria, Australia

${ }^{3}$ School of Cancer Medicine and Molecular Cancer Prevention Program, La Trobe University, Melbourne, Victoria, Australia ${ }^{4}$ Department of Clinical Pathology, The University of Melbourne, Parkville, Victoria, Australia

${ }^{5}$ Department of Surgery, The University of Melbourne, Heidelberg, Victoria, Australia ${ }^{6}$ Sydney Medical School, The University of Sydney, Camperdown, New South Wales, Australia

${ }^{7}$ School of Medicine, Western Sydney University, Sydney, New South Wales, Australia

\section{Correspondence to}

Dr Andrew J Colebatch, Tissue Pathology and Diagnostic Oncology, Royal Prince Alfred Hospital, Sydney, Australia; andrew.colebatch@health.nsw. gov.au

Received 12 December 2018 Revised 3 January 2019 Accepted 4 January 2019 Published Online First 29 January 2019
Check for updates

(C) Author(s) (or their employer(s)) 2019. No commercial re-use. See rights and permissions. Published by BMJ.

To cite: Colebatch $A J$,

Dobrovic A, Cooper WA.

J Clin Pathol

2019:72:281-284

\section{ABSTRACT}

In this review, we summarise the function and structure of telomerase reverse transcriptase (TERT) in humans, including its regulation. The dysregulation of telomerase through TERT promoter mutations across a range of cancers is discussed. The molecular mechanism activated by TERT promoter mutations is outlined. Finally, the timing of TERT promoter mutations during carcinogenesis is reviewed in the context of their potential utility as clinical biomarkers of malignant transformation.

\section{INTRODUCTION}

The immortalisation of cancer cells is a critical hallmark required for malignant behaviour. ${ }^{1}$ The reactivation of telomerase by tumours is the most common pathway contributing to the immortalised state, and in turn the most common somatic event to achieve this involves mutations in the promoter of telomerase reverse transcriptase (TERT), the catalytic subunit of telomerase. By contrast, deleterious germline TERT mutations have been shown to be associated with dyskeratosis congenita (a progeria) and pulmonary fibrosis. As a result, there is interest in understanding TERT, its regulation and dysregulation. More specifically, elucidating the molecular mechanism of how promoter mutations in TERT lead to increased expression of telomerase during carcinogenesis and how these alterations could potentially be exploited for diagnostic and therapeutic purposes is of great interest to researchers and clinicians.

\section{The structure and function of TERT}

The linear organisation of eukaryotic chromosomes results in two fundamental problems: first, the ends of the chromosomes being recognised as double-stranded breaks by the cell, and second the erosion of the 5' end during cycles of genomic replication due to non-reproduction of the RNA primer binding site. ${ }^{2}$

The former problem is avoided by the presence of telomeres at the ends of chromosomes. Telomeres are tandem repeats of TTAGGG (in vertebrates and most metazoans ${ }^{3}$ ) which, when assembled with a protein complex known as shelterin, protect the ends of chromosomes from DNA repair pathways initiated by a DNA damage response.

The latter problem, known as the 'end replication problem', can be rectified by telomerase. The ribonucleoprotein telomerase has a core component composed of a reverse transcriptase (TERT) subunit and a separately coded RNA template (TERC or TR), which, along with a series of associate proteins, leads to the extension and replenishment of telomeres.
In humans, telomerase is normally expressed during development in embryonic stem cells and later silenced in somatic cells on differentiation. ${ }^{4}$ Telomerase expression is maintained to a restricted set of cells, primarily transit-amplifying stem-like cells and germ cells.

In the absence of telomere expansion, the proliferative capacity of human cells is limited by the continuous erosion of telomeres, leading to cellular senescence. The number of cellular divisions prior to senescence is known as the Hayflick limit. ${ }^{5}$ This senescence response is a critical tumour suppressive mechanism, and yet can be the source of various diseases as the result of short telomeres, such as lung fibrosis. ${ }^{6}$

\section{The role of TERT in cancer}

Telomerase is detectable in over $90 \%$ of human cancers, ${ }^{47}$ and its re-expression is thought to represent the most common pathway to cellular immortalisation. It has been shown that the increased expression of TERT leads to restoration of telomerase activity, ${ }^{89}$ thereby implicating the transcriptional control of TERT as a key factor in diseases, including ageing and cancer.

During neoplastic progression, cellular senescence is bypassed by the inactivation of cell cycle checkpoint genes such as p53 and pRb. ${ }^{10}$ With continuing cycles of cellular division past the point of replicative senescence (M1 stage), telomeres become critically shortened and cells enter 'crisis' (M2 stage). In crisis, chromosome end fusions and breakage-fusion-bridge events occur, leading to chromosomal instability. Cells escape crisis through either upregulation of telomerase or the alternate lengthening of telomeres mechanism. ${ }^{11}$ In the latter process, telomeres are replenished using homologous recombination.

TERT is located on chromosome 5 and consists of 16 exons. ${ }^{12} 13$ The protein product TERT exists as a dimer within the telomerase holoenzyme ${ }^{14}$ and consists of three regions: (1) the N-terminal extension that contains an N-terminal domain and a telomerase RNA-binding domain; (2) the central catalytic reverse transcriptase domain; and (3) the C-terminal extension. ${ }^{15}$ This basic organisation has remained remarkably stable over evolutionary time ${ }^{16}$ implying that it was present in basal metazoans at the emergence of multicellularity. Interestingly, TERT is not present in Drosophila, as this genus does not use telomerase to solve the end replication problem. ${ }^{17}$

The promoter region of TERT has been extensively studied in order to identify the candidate factors involved in influencing TERT expression. (For a comprehensive review of the promoter structure and binding partners, see ref 18.) The TERT 
promoter is guanine-cytosine rich (GC-rich)and lacks both a TATA box and a CAAT box. Multiple factors have been shown to influence TERT expression in context-specific ways, either singly or in coordination, including Myc, oestrogen, SP1, nuclear factor kappa B (NF-kB), p53, activator protein 1 (AP-1) and E2F factors. Recognition sequences for binding of p53, p21, SP1, E26 transformation-specific (ETS), E2F, AP-1, hypoxia-inducregion. ${ }^{18}$ Interestingly, the ETS family member gene GABPA, which binds TERT promoter mutations in cancer (described below), does not appear to bind the native promoter. ${ }^{19}$

\section{Splice variants of TERT}

While the transcriptional regulation of TERT has been studied in depth, recent work has evaluated the role of alternate splicing of mRNA transcripts. TERT can be translated from multiple differently spliced transcripts, with only the longest variant having reverse transcriptase enzymatic activity. ${ }^{20}$ Breast cancer cell lines with overexpression of transcripts without catalytic function have been shown to have reduced apoptosis, conferring a survival advantage. ${ }^{21}$ This suggests novel functions of TERT beyond telomere extension.

\section{TERT upregulation in cancer/TERT promoter mutations}

TERT can be upregulated in cancer through several mechanisms, including gene amplification, rearrangements and mutations within its promoter. TERT has been shown to be amplified in approximately $4 \%$ of cancers, especially ovarian cancer, lung adenocarcinoma, lung squamous cell carcinoma, oesophageal carcinoma and adrenocortical carcinoma. ${ }^{22}$ Rearrangements of the TERT promoter have been demonstrated in high-risk neuroTERT appear to be relatively rare overall in cancer, although common in specific subtypes. ${ }^{22}$ ible factor 1 (HIF1) and c-myc are present within the promoter blastomas. ${ }^{22}{ }^{23}$ However, amplifications and rearrangements of

The identification of frequent non-coding TERT promoter mutations in cutaneous melanoma ${ }^{24} 25$ at once simultaneously delineated a novel class of cancer driver mutations, as well as indicated the mechanism by which TERT upregulation occurred in a large proportion of tumours. These mutations are recurrent $\mathrm{C}>\mathrm{T}$ transitions occurring at chr5:1295228 ( -124 or C228T) or chr5:1295250 ( -146 or C250T) (hg19/GCRh37 genome coordinates) within the core promoter of TERT, leading to the creation of novel ETS transcription factor binding sites. Initial studies using a luciferase reporter assay demonstrated increased TERT gene expression as a result of these mutations. ${ }^{24}$

TERT promoter mutations were subsequently demonstrated across a range of malignancies. Of note, the frequency of TERT promoter mutations varies considerably with tumour subtype. In melanoma, up to $80 \%$ of non-acral cutaneous melanomas have been shown to have TERT promoter mutations, ${ }^{26-29}$ while less than $10 \%$ of acral melanomas ${ }^{30}$ and less than $1 \%$ of uveal melanomas ${ }^{31}$ have these mutations. In tumours of the central nervous system, TERT promoter mutations are present in approximately $70 \%$ of primary glioblastomas and oligodendrogliomas, but are relatively infrequent in secondary glioblastomas. $^{32-35}$ TERT promoter mutations are also common in urothelial carcinoma, ${ }^{36} 37$ poorly differentiated and anaplastic thyroid carcinomas, ${ }^{38} 39$ cutaneous basal cell and squamous cell carcinomas, ${ }^{40} 41$ and hepatocellular carcinoma ${ }^{42}$ (comprehensively and recently reviewed in ref 43 ).

The mutations in C228T and C250T, which rarely co-occur, ${ }^{26} 27$ both lead to the creation of an identical 11-base sequence which corresponds to a de novo binding site for an ETS transcription factor (figure 1). Reporter assays have demonstrated that both C228T and C250T lead to increased transcription of TERT, ${ }^{24} 25$ approximately doubling wild-type promoter expression. Increased telomerase expression has also been demonstrated in urothelial carcinomas with TERT promoter

\section{Novel ETS binding site}
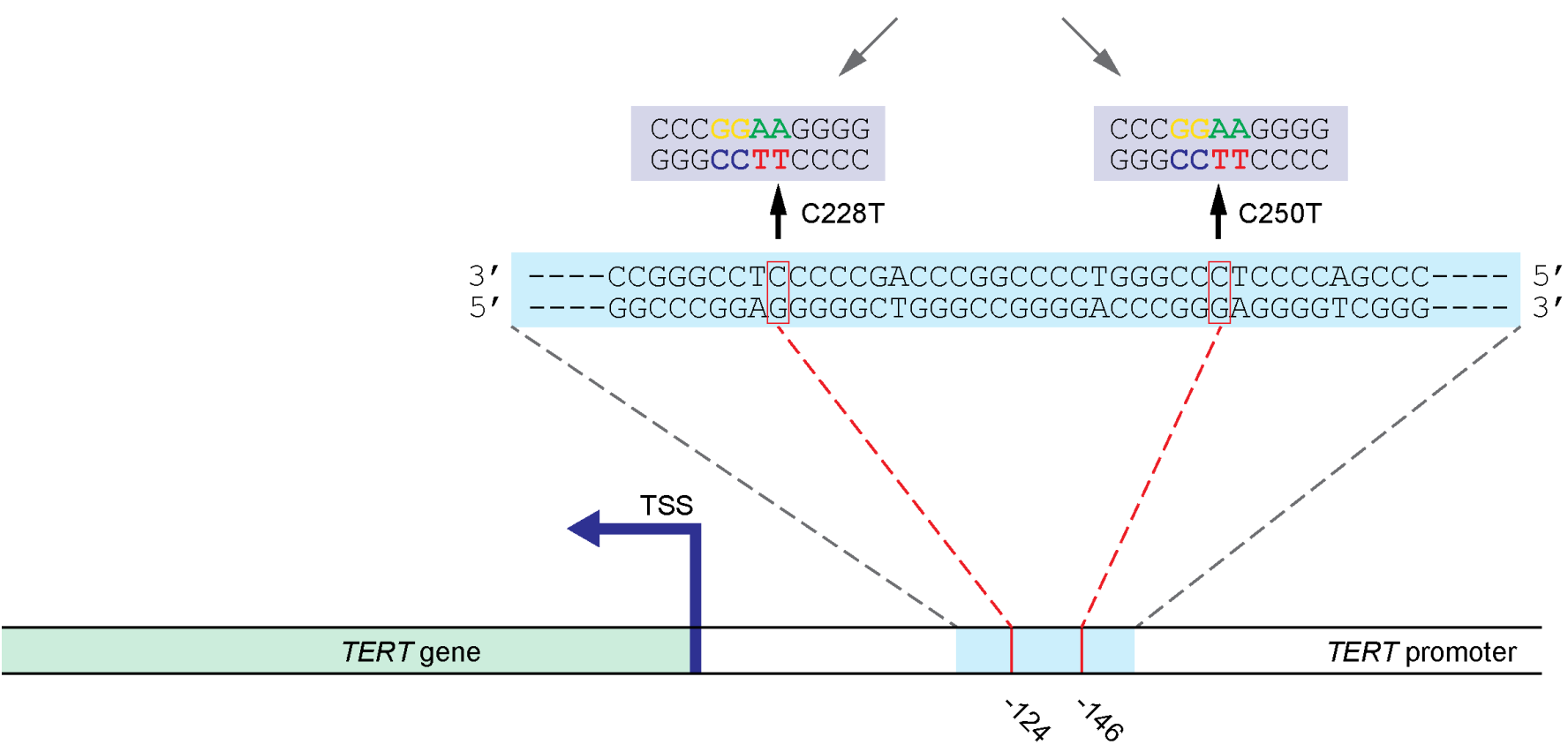

Figure 1 TERT promoter mutations occur most commonly at two positions. Two $C \rightarrow$ T mutations located at -124 and -146 base pairs from the TSS within the core promoter of TERT account for the majority of TERT promoter mutations identified in cancer. These mutations lead to the creation of novel ETS transcription factor binding sites which drive increased expression of TERT. ETS, E26 transformation-specific; TERT, telomerase reverse transcriptase; TSS, transcriptional start site. 
mutations. ${ }^{36}$ Additionally, TERT promoter mutations are associated with switching of inactive to active chromatin marks in the TERT promoter. ${ }^{44}$ The likely ETS factor responsible for increased expression has been identified as GABPA, ${ }^{45}$ which is the only one of the 26 members of the ETS family that forms multimeric complexes when driving gene expression. A recent study examining glioblastoma cell lines found GABP $\beta 1 \mathrm{~L}$ to be the critical binding partner of GABPA in driving TERT expression in the presence of a TERT promoter mutation. ${ }^{19}$ Another group has suggested that the $\mathrm{C} 228 \mathrm{~T}$ and $\mathrm{C} 250 \mathrm{~T}$ mutations are functionally distinct: the former leading to GABPA recruitment; the latter leading to the generation of both an ETS site and a functional p52 site requiring ETS1/2 as the culpable ETS factor. ${ }^{46}$

The mechanism by which TERT promoter mutations ultimately facilitate cancer growth is still being elucidated. Whereas the initial studies used reporter assays to demonstrate increased TERT expression, the demonstrated increase in TERT expression appears to be relatively modest, and several studies have failed to find a significant association between TERT promoter status and TERT expression. ${ }^{28} 3647$ However, modest expression of TERT may still be sufficient for tumour cells to have a selective advantage. ${ }^{48}$ Comparison between matched naevi and adjacent melanoma demonstrates increased TERT expression in the malignant component with a TERT promoter mutation. ${ }^{49} \mathrm{~A}$ recent study by Chiba $e t a l^{50}$ demonstrated that, by contrast, telomere length was shorter in the melanomas than in the adjacent naevi. ${ }^{50} \mathrm{In}$ this latter study, the investigators propose that TERT promoter mutations act in two stages, with the first phase having moderately increased TERT expression yet at a level insufficient to prevent telomere shortening, resulting in shorter telomeres and delayed replicative senescence. In the second phase, there is telomere-driven genomic instability and upregulated telomerase activity.

A recent systematic pan-cancer analysis of more than 2500 tumour genomes found very few potential recurrent non-coding driver point mutations aside from those in TERT. ${ }^{51}$ The pan-cancer analysis confirmed earlier results which did not find other non-coding mutations in cancer leading to changes in gene expression. ${ }^{47}$ Rather than being an exemplar of a potential novel class of non-coding driver mutations, TERT promoter mutations appear to be unique, likely a result of the peculiar biology of telomerase and its regulation.

\section{Timing of TERT promoter mutations and their use as a biomarker}

While TERT promoter mutations demonstrate that the cells of origin of specific tumours have had downregulated TERT at some point, it is not clear at what point during tumourigenesis these mutations are acquired. Given that the selective pressure for TERT promoter mutations should be strongest near the Hayflick limit after multiple rounds of replication, ${ }^{5}$ and therefore relatively late, it is somewhat unexpected that TERT promoter mutations are early events in carcinogenesis. Indeed, accumulating evidence suggests that TERT promoter mutations, at least in some tumours, are not an initiating event, but are selected for early in neoplasia, at an intermediate, preinvasive phenotype. ${ }^{52-54}$ As such, TERT promoter mutations are potentially a useful diagnostic biomarker, as these lesions can potentially be treated with curative intent. However, in certain lesions, TERT promoter mutation detection will not distinguish diagnostically between malignant and intermediate entities.

The promise of the use of TERT promoter mutations as a clinical biomarker for cancer are threefold: first, that these mutations appear as a manifestation of a specific hallmark of cancer (although not fully tested yet in benign tissue) ${ }^{1}$; second, that these occur early in tumour development ${ }^{4952-55}$; and third, that these mutations are recurrent in relatively few genomic positions (with the two canonical -124 and -146 point mutations accounting for over $85 \%$ of described mutations) in a range of malignancies. ${ }^{43} 55$

TERT promoter mutations have multiple potential uses as clinical biomarkers. TERT promoter mutations can be detected in urine samples from patients with recurrent urothelial carcinoma. ${ }^{56}$ In melanoma, several approaches using droplet digital PCR have been recently developed ${ }^{57} 58$ which can monitor TERT promoter mutations in serum. Moreover, TERT promoter mutation detection indicates a worse prognosis in thyroid malignancies ${ }^{59}$ melanoma ${ }^{27}$ and gliomas. ${ }^{6061}$

\section{TERT in conditions other than cancer}

Germline mutations in TERT have been associated with idiopathic pulmonary fibrosis, ${ }^{62}$ and rare cases of germline mutations in TERT have been shown in families with dyskeratosis congenita. ${ }^{63}$ TERT mutations have also been found in patients with severe emphysema. ${ }^{64}$ Interestingly, somatic mutations in the TERT promoter have been found in the leucocytes of some individuals with germline mutations in TERT, suggesting that these mutations act as counterbalances to one another regarding TERT expression. ${ }^{65}$

\section{CONCLUSION}

The TERT gene plays important roles in normal biology, and perturbations of its regulation play a critical role in a variety of pathological states, especially neoplasia. In particular, TERT plays a central role in modulating telomerase activity in tumours, conferring the hallmark of immortality on neoplastic clones. A deeper understanding of this gene is pertinent given its potential usage as a biomarker and the future development of possible therapeutic avenues.

\section{Handling editor Des Richardson.}

Contributors AJC was the main author. All authors contributed to the conception of the work and revising it critically, and approved the final version.

Funding The authors have not declared a specific grant for this research from any funding agency in the public, commercial or not-for-profit sectors.

Competing interests None declared.

Patient consent Not required.

Provenance and peer review Commissioned; externally peer reviewed.

\section{REFERENCES}

1 Hanahan D, Weinberg RA. Hallmarks of cancer: the next generation. Cell 2011;144:646-74.

2 Henderson ER, Blackburn EH. An overhanging $3^{\prime}$ terminus is a conserved feature of telomeres. Mol Cell Biol 1989:9:345-8.

3 Gomes NM, Shay JW, Wright WE. Telomere biology in Metazoa. FEBS Lett 2010:584:3741-51.

4 Kim NW, Piatyszek MA, Prowse KR, et al. Specific association of human telomerase activity with immortal cells and cancer. Science 1994;266:2011-5.

5 Hayflick L, Moorhead PS. The serial cultivation of human diploid cell strains. Exp Cell Res 1961;25:585-621.

6 Tsakiri KD, Cronkhite JT, Kuan PJ, et al. Adult-onset pulmonary fibrosis caused by mutations in telomerase. Proc Natl Acad Sci U S A 2007;104:7552-7.

7 Shay JW, Bacchetti S. A survey of telomerase activity in human cancer. Eur J Cancer 1997;33:787-91

8 Nakamura TM, Morin GB, Chapman KB, et al. Telomerase catalytic subunit homologs from fission yeast and human. Science 1997;277:955-9.

9 Counter CM, Meyerson M, Eaton EN, et al. Telomerase activity is restored in human cells by ectopic expression of hTERT (hEST2), the catalytic subunit of telomerase. Oncogene 1998;16:1217-22. 
10 Shay JW, Wright WE, Werbin H. Defining the molecular mechanisms of human cell immortalization. Biochimica et Biophysica Acta (BBA) - Reviews on Cancer 1991;1072:1-7.

11 Dunham MA, Neumann AA, Fasching CL, et al. Telomere maintenance by recombination in human cells. Nat Genet 2000;26:447-50.

12 Cong YS, Wen J, Bacchetti S. The human telomerase catalytic subunit hTERT: organization of the gene and characterization of the promoter. Hum Mol Genet 1999:8:137-42.

13 Wick M, Zubov D, Hagen G. Genomic organization and promoter characterization of the gene encoding the human telomerase reverse transcriptase (hTERT). Gene 1999;232:97-106.

14 Sauerwald A, Sandin S, Cristofari G, et al. Structure of active dimeric human telomerase. Nat Struct Mol Biol 2013;20:454-60.

15 Wyatt HD, West SC, Beattie TL. InTERTpreting telomerase structure and function. Nucleic Acids Res 2010;38:5609-22.

16 Lai AG, Pouchkina-Stantcheva N, Di Donfrancesco A, et al. The protein subunit of telomerase displays patterns of dynamic evolution and conservation across different metazoan taxa. BMC Evolutionary Biology 2017;17:107.

17 Villasante A, Abad JP, Planelló R, et al. Drosophila telomeric retrotransposons derived from an ancestral element that was recruited to replace telomerase. Genome Res 2007;17:1909-18.

18 Akincilar SC, Unal B, Tergaonkar V. Reactivation of telomerase in cancer. Cell Mol Life Sci 2016;73:1659-70.

19 Mancini A, Xavier-Magalhães A, Woods WS, et al. Disruption of the $\beta 1 \mathrm{~L}$ isoform of $\mathrm{GABP}$ reverses glioblastoma replicative immortality in a TERT promoter mutationdependent manner. Cancer Cell 2018:34:513-28.

20 Saebøe-Larssen S, Fossberg E, Gaudernack G. Characterization of novel alternative splicing sites in human telomerase reverse transcriptase (hTERT): analysis of expression and mutual correlation in mRNA isoforms from normal and tumour tissues. BMC Mol Biol 2006:7:26

21 Listerman I, Sun J, Gazzaniga FS, et al. The major reverse transcriptase-incompetent splice variant of the human telomerase protein inhibits telomerase activity but protects from apoptosis. Cancer Res 2013;73:2817-28.

22 Barthel FP, Wei W, Tang M, et al. Systematic analysis of telomere length and somatic alterations in 31 cancer types. Nat Genet 2017:49:349-57.

23 Peifer M, Hertwig F, Roels F, et al. Telomerase activation by genomic rearrangements in high-risk neuroblastoma. Nature 2015;526:700-4.

24 Huang FW, Hodis E, Xu MJ, et al. Highly recurrent TERT promoter mutations in human melanoma. Science 2013:339:957-9.

25 Horn S, Figl A, Rachakonda PS, et al. TERT promoter mutations in familial and sporadic melanoma. Science 2013;339:959-61.

26 Heidenreich B, Nagore E, Rachakonda PS, et al. Telomerase reverse transcriptase promoter mutations in primary cutaneous melanoma. Nat Commun 2014:5:3401.

27 Griewank KG, Murali R, Puig-Butille JA, et al. TERT promoter mutation status as an independent prognostic factor in cutaneous melanoma. Journal of the National Cancer Institute 2014;106.

28 Akbani R, Akdemir KC, Aksoy BA, et al. Genomic classification of cutaneous melanoma. Cell 2015;161:1681-96.

29 Ekedahl $\mathrm{H}$, Lauss $\mathrm{M}$, Olsson $\mathrm{H}$, et al. High TERT promoter mutation frequency in non-acral cutaneous metastatic melanoma. Pigment Cell Melanoma Res 2016;29:598-600

30 Liau JY, Tsai JH, Jeng YM, et al. TERT promoter mutation is uncommon in acral lentiginous melanoma. J Cutan Pathol 2014;41:504-8.

31 Koopmans AE, Ober K, Dubbink HJ, et al. Prevalence and implications of TERT promoter mutation in uveal and conjunctival melanoma and in benign and premalignant conjunctival melanocytic lesions. Invest Ophthalmo/ Vis SC 2014:55:6024-30.

32 Eckel-Passow JE, Lachance DH, Molinaro AM, et al. Glioma groups based on 1p/19q, IDH, and TERT promoter mutations in tumors. N Engl J Med 2015:372:2499-508.

33 Nonoguchi N, Ohta T, Oh JE, et al. TERT promoter mutations in primary and secondary glioblastomas. Acta Neuropathol 2013;126:931-7.

34 Killela PJ, Reitman ZJ, Jiao Y, et al. TERT promoter mutations occur frequently in gliomas and a subset of tumors derived from cells with low rates of self-renewal. Proceedings of the National Academy of Sciences 2013;110:6021-6.

35 Arita $\mathrm{H}$, Narita Y, Fukushima $\mathrm{S}$, et al. Upregulating mutations in the TERT promoter commonly occur in adult malignant gliomas and are strongly associated with total 1 19q loss. Acta Neuropathol 2013;126:267-76.

36 Borah S, Xi L, Zaug AJ, et al. Cancer. TERT promoter mutations and telomerase reactivation in urothelial cancer. Science 2015;347:1006-10.

37 Allory Y, Beukers W, Sagrera A, et al. Telomerase reverse transcriptase promoter mutations in bladder cancer: high frequency across stages, detection in urine, and lack of association with outcome. Eur Urol 2014;65:360-6.
38 Liu X, Bishop J, Shan Y, et al. Highly prevalent TERT promoter mutations in aggressive thyroid cancers. Endocr Relat Cancer 2013;20:603-10.

39 Landa I, Ganly I, Chan TA, et al. Frequent somatic TERT promoter mutations in thyroid cancer: higher prevalence in advanced forms of the disease. J Clin Endocrinol Metab 2013;98:E1562-E1566

40 Griewank KG, Murali R, Schilling B, et al. TERT promoter mutations are frequent in cutaneous basal cell carcinoma and squamous cell carcinoma. PLoS One 2013:8:e80354.

41 Scott GA, Laughlin TS, Rothberg PG. Mutations of the TERT promoter are common in basal cell carcinoma and squamous cell carcinoma. Mod Pathol 2014;27:516-23.

42 Nault JC, Mallet M, Pilati C, et al. High frequency of telomerase reverse-transcriptase promoter somatic mutations in hepatocellular carcinoma and preneoplastic lesions. Nat Commun 2013:4:2218

43 Gaspar TB, Sa A, Lopes JM, et al. Telomere maintenance mechanisms in cancer. Genes 2018;9:241

44 Stern JL, Theodorescu D, Vogelstein B, et al. Mutation of the TERT promoter, switch to active chromatin, and monoallelic TERT expression in multiple cancers. Genes Dev 2015;29:2219-24.

45 Bell RJ, Rube HT, Kreig A, et al. Cancer. the transcription factor GABP selectively binds and activates the mutant TERT promoter in cancer. Science 2015;348:1036-9.

46 Li Y, Zhou QL, Sun W, et al. Non-canonical NF- $\kappa B$ signalling and ETS1/2 cooperatively drive C250T mutant TERT promoter activation. Nat Cell Biol 2015;17:1327-38.

47 Fredriksson NJ, Ny L, Nilsson JA, et al. Systematic analysis of noncoding somatic mutations and gene expression alterations across 14 tumor types. Nat Genet 2014;46:1258-63.

48 Cui W, Aslam S, Fletcher J, et al. Stabilization of telomere length and karyotypic stability are directly correlated with the level of hTERT gene expression in primary fibroblasts. J Biol Chem 2002;277:38531-9.

49 Shain AH, Joseph NM, Yu R, et al. Genomic and transcriptomic analysis reveals incremental disruption of key signaling pathways during melanoma evolution. Cancer Cell 2018;34:45-55.

50 Chiba K, Lorbeer FK, Shain AH, et al. Mutations in the promoter of the telomerase gene TERT contribute to tumorigenesis by a two-step mechanism. Science 2017;357:1416-20.

51 Rheinbay E, Nielsen MM, Abascal F, et al. Discovery and characterization of coding andnon-coding driver mutations in more than 2,500 whole cancer genomes. BioRxiv 2017.

52 Shain $\mathrm{AH}$, Yeh I, Kovalyshyn I, et al. The genetic evolution of melanoma from precursor lesions. N Engl J Med 2015:373:1926-36.

53 Torrecilla S, Sia D, Harrington AN, et al. Trunk mutational events present minimal intra- and inter-tumoral heterogeneity in hepatocellular carcinoma. $J$ Hepatol 2017:67:1222-31

54 Rudini N, Novello C, Destro A, et al. Phenotypic and molecular changes in nodulein-nodule hepatocellular carcinoma with pathogenetic implications. Histopathology 2018;73:601-11.

55 Bell RJ, Rube HT, Xavier-Magalhães A, et al. Understanding TERT promoter mutations: a common path to immortality. Mol Cancer Res 2016;14:315-23.

56 Papadopoulos N, Kinde I, Munari E. TERT promoter mutations occur early in urothelia neoplasia and are biomarkers of early disease and disease recurrence in urine. Cancer research 2013.

57 McEvoy AC, Calapre L, Pereira MR, et al. Sensitive droplet digital PCR method for detection of TERT promoter mutations in cell free DNA from patients with metastatic melanoma. Oncotarget 2017;8:78890-900.

58 Colebatch AJ, Witkowski T, Waring PM, et al. Optimizing Amplification of the GC-Rich TERT Promoter Region Using 7-Deaza-dGTP for Droplet Digital PCR Quantification of TERT Promoter Mutations. Clin Chem 2018;64:745-7.

$59 \operatorname{Kim} \mathrm{TH}, \mathrm{Kim}$ YE, Ahn S, et al. TERT promoter mutations and long-term survival in patients with thyroid cancer. Endocr Relat Cancer 2016;23:813-23.

60 Chan AK, Yao Y, Zhang Z, et al. TERT promoter mutations contribute to subset prognostication of lower-grade gliomas. Mod Pathol 2015;28:177-86.

61 Batista R, Cruvinel-Carloni A, Vinagre J, et al. The prognostic impact of TERT promoter mutations in glioblastomas is modified by the rs 2853669 single nucleotide polymorphism. Int J Cancer 2016;139:414-23.

62 Armanios MY, Chen JJ, Cogan JD, et al. Telomerase mutations in families with idiopathic pulmonary fibrosis. N Engl J Med 2007;356:1317-26.

63 Armanios M, Chen J-L, Chang Y-PC, et al. Haploinsufficiency of telomerase reverse transcriptase leads to anticipation in autosomal dominant dyskeratosis congenita. Proceedings of the National Academy of Sciences 2005:102:15960-4.

64 Stanley SE, Chen JJ, Podlevsky JD, et al. Telomerase mutations in smokers with severe emphysema. J Clin Invest 2015:125:563-70.

65 Maryoung L, Yue Y, Young A, et al. Somatic mutations in telomerase promoter counterbalance germline loss-of-function mutations. J Clin Invest 2017;127:982-6. 